\title{
Estratégias de cuidado interprofissional na assistência a saúde da puérpera na atencão primária à saúde. Uma revisão integrativa sobre prática clínica*
}

\section{Estrategias de cuidado interprofesional en la asistencia a la salud de la puérpera. Una revisión integrativa sobre la práctica clínica}

\section{Interprofessional care strategies in postpartum health care. An integrative review of clinical practice}

Recepção: 15 de novembro de 2019. Aprovação: 08 de julho de 2020. Publicação: 30 de junho de 2021.

DOI: https://doi.org/10.11144/Javeriana.rgps20.ecia

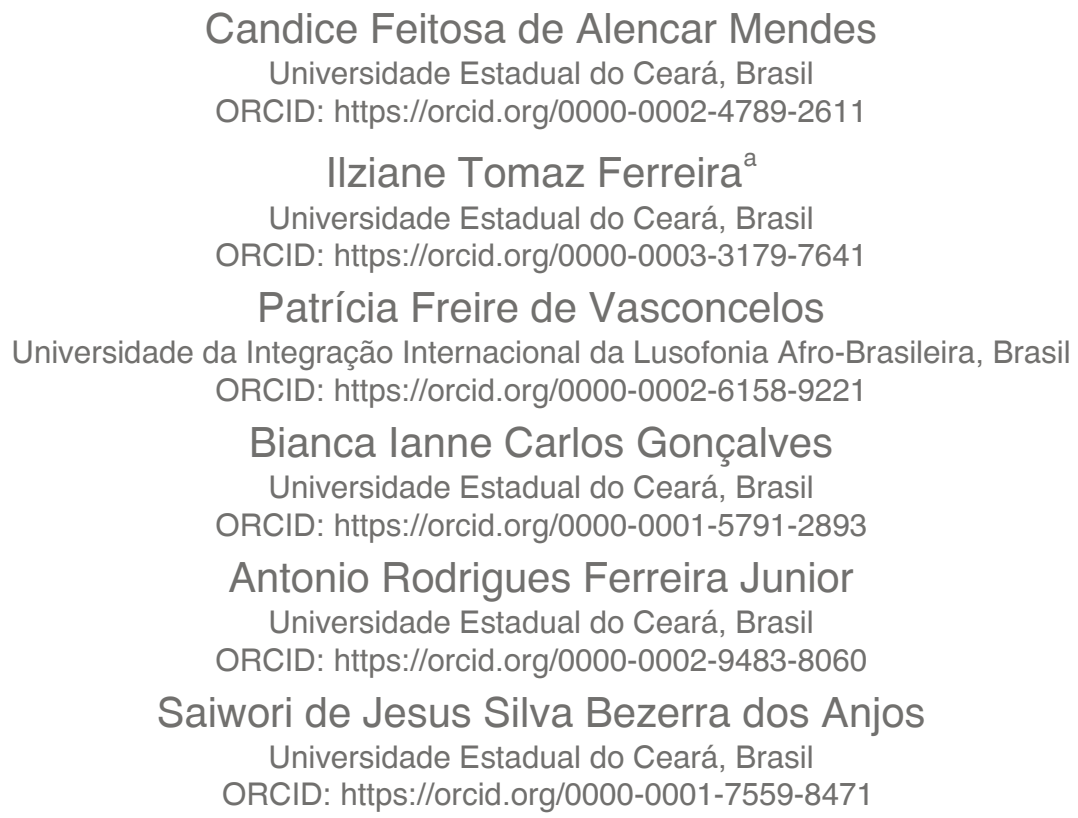

Para citar este artigo: Mendes CFA, Ferreira IT, Vasconcelos BIC, Gonçalves BIC, Ferreira Jr AR, Anjos BSJS. Estratégias de cuidado interprofissional na assistência a saúde da puérpera na atenção primária à saúde. Uma revisão integrativa sobre prática clínica. Rev Gerenc Polit Salud. 2021;20. https://doi.org/10.11144/ Javeriana.rgps20.ecia

* Artículo de investigación.

a Autora de correspondencia. Correo electrónico: ilzianne@hotmail.com 


\section{Resumo}

Introdução. A Atenção Primária à Saúde visa a promoção da saúde materna, e a prevenção e tratamento de complicações que possam ocorrer no período gravídico e puerperal. Objetivo. Analisar a literatura acerca do cuidado multiprofissional à puérpera na Atenção Primária à Saúde. Método. Foram realizadas buscas com os descritores em ciência da saúde (Equipe de Assistência ao Paciente, Período Pós-Parto e Atenção Primária à Saúde), nas seguintes bases de dados: Literatura Latino-Americana e do Caribe em Ciências da Saúde (LILACS), Embase, Web of Science, Cochrane Library e Medline. Foram incluídos na integra artigos publicados e indexados nas referidas bases que abordassem o cuidado às puérperas. Resultados. A análise da síntese quantitativa compreendeu-se em 12 publicações. Os cuidados às puérperas apresentados foram: visita domiciliar, assistência médica, assistência psicológica, educação em saúde, assistência nutricional e acolhimento. Conclusão. Para fortalecer os conhecimentos e as práticas de cuidado neste período, cada profissional deve reconhecer o cenário de apoio necessário ao binômio mãe-filho. Assim, o uso da visita domiciliar como instrumento primordial deve ser o campo de atuação de toda a equipe, desenvolvendo uma colaboração interprofissional, e contextualizando os aspectos físico e mental da saúde.

Palavras-chave: Equipe de assistência ao paciente, período pós-parto, atenção primária à saúde.

\section{Resumen}

Introducción. La Atención Primaria en Salud contempla la promoción de la salud materna, prevención y tratamiento de complicaciones que puedan ocurrir en el periodo de gestación y puerperal. Objetivo. Analizar la literatura del cuidado multiprofesional de la puérpera en la Atención Primaria en Salud. Método. Se realizaron búsquedas con los descriptores en ciencias de la salud (equipo de asistencia al paciente, periodo posparto, atención primaria en salud) en las siguientes bases de datos: Literatura Latinoamericana y del Caribe en Ciencias de la Salud (LILACS), Embase, Web of Science, Cochrane Library y Medline. Se incluyeron todos los artículos publicados e indexados que abordan el cuidado de las puérperas en las bases referidas. Resultados. El análisis de la síntesis cuantitativa comprende 12 publicaciones. Los cuidados de las puérperas presentados fueron: visita domiciliar, asistencia médica, asistencia psicológica, educación en salud, asistencia nutricional y acogida. Conclusión. Para fortalecer los conocimientos y las prácticas de cuidado en este periodo cada profesional debe reconocer el escenario de apoyo necesario del binomio madre-hijo. Así, el uso de la visita domiciliar como instrumento primordial debe ser un campo de actuación de todo el equipo, desarrollando una colaboración interprofesional, y contextualizando los aspectos físico y mental de la salud.

Palabras clave: Equipo de atención al paciente, periodo pospart, atención primaria en salud, periodo posparto.

\section{Abstract}

Introduction. Primary Health Care aims to promote maternal health, prevention and treatment of complications during gestation and the postpartum period. Objective. To analyze the literature relating to multi-professional postpartum care in Primary Health Care. Methods. Searches were done with health science descriptors (Patient Assistant Team, Postpartum Period and Primary Health Care), in the following databases: Latin American and Caribbean Health Sciences (LILACS), Embase, Web of Science, Cochrane Library and Medline. Published and indexed articles of these databases relating to postpartum care were included. Results. Quantitative synthetic analyses include 12 publications. The following postpartum care services were observed: home visits, medical assistance, psychological assistance, health education, nutritional assistance, and accommodation. Conclusion. To strengthen the knowledge and practices of care during this period, professionals must identify the support scenario for the mother-child binomial. Thus, using home visits as a primary instrument must be a field of action for the whole team. They must develop an interprofessional collaboration and a contextualized approach to the physical and mental aspects of health.

Keywords: Patient care team, postpartum period, primary health care. 


\section{Introdução}

A atenção à saúde da mulher tem investido na formulação e implementação de políticas de promoção, proteção e recuperação da saúde dessa população, priorizando ações de melhoria e ampliação da qualidade de vida. Esse contexto, compreende o espaço fundamental para fomentar mudanças no núcleo do cuidado à mulher, principalmente com vistas a acolhê-la no período da gestação, parto e puerpério, garantindo o direito a uma atenção de qualidade, como componente da cidadania (1).

Apesar da redução mundial das taxas de mortalidade durante a gravidez e o parto, ainda se observam índices elevados nos países em desenvolvimento, visto que $99 \%$ dos óbitos maternos ocorrem nesses países. Os casos de óbitos que são considerados evitáveis continuam acontecendo em proporções alarmantes, sobretudo decorrente de doenças hipertensivas, hemorragias, sepse/infecções e complicações relacionadas ao aborto. Ainda, ressalta-se que a maioria dos óbitos maternos se concentra no puerpério imediato ( $1^{\circ}$ ao $10^{\circ}$ dia pós-parto), além de ser considerado uma fase de morbidade significativa que se estende para o puerpério tardio (do $11^{\circ}$ ao $45^{\circ}$ dia) e remoto (após $45^{\circ}$ dia), com estudos que apontam para agravos persistindo por mais de 4 anos pós-parto (2).

A Atenção Primária em Saúde (APS), especialmente a Estratégia de Saúde da Família (ESF), visa à promoção da saúde materna, prevenção e tratamento de complicações que possam ocorrer no período gravídico e puerperal. A ESF possui sua essência centrada no cuidado, na escuta qualificada e no elo entre profissional-usuário. A atenção à gestante, ao cuidado pré-natal e à assistência puerperal são características específicas do processo de trabalho dessa estratégia. Estas conferem maior proximidade com as gestantes, realizando atividades desenvolvidas nas unidades de saúde como nos domicílios (3).

Nesse cenário, a APS torna-se coordenadora da atenção à mulher no pós-parto, por meio da construção do conhecimento técnico e da capacidade de acolher, apoiar e detectar mudanças físicas e emocionais precocemente. Igualmente, a APS presta ações de prevenção, tratamento e acompanhamento da mulher, com intervenções e encaminhamentos para outros serviços especializados (4).

É a partir da associação e trabalho mútuo entre diversos profissionais da saúde que se constrói a disseminação de diferentes olhares sobre as práticas do cuidado à saúde materno-infantil, garantindo uma atenção integral e ampliando o potencial de resolutividade na APS. Nesse campo de ação da atenção, apesar dos avanços no Brasil e no mundo, a assistência ao puerpério ainda é um fator preocupante na saúde pública e requer uma atenção diferenciada (5).

Nessa perspectiva, o estudo tem como objetivo analisar as evidências científicas acerca do cuidado multiprofissional à puérpera na APS, com a finalidade de evidenciar o contexto da assistência à saúde da mulher, possibilitando qualificar intervenções nesses lócus de atuação. 
Candice Feitosa de Alencar Mendes/ Ilziane Tomaz Ferreira/ Patrícia

Freire de Vasconcelos/ Bianca Ianne Carlos Gonçalves/ et ai.

\section{Metodologia}

Trata-se de uma revisão integrativa da literatura, realizada por meio das seguintes etapas: identificação da questão norteadora do estudo; busca na literatura; avaliação dos artigos, a partir dos seguintes passos: título, resumo, abordagem metodológica, leitura na íntegra e avaliação de qualidade dos estudos a partir do formulário padronizado Critical Appraisal Skills Programme (CASP) (6).

Foi utilizada a estratégia PICo ( $\mathrm{P}=$ Mulher no puerpério; $\mathrm{I}=$ Acompanhamento interprofissional (práticas/condutas/ações); $\mathrm{Co}=$ Protocolos clínicos de atenção às puérperas na APS) para elaboração da questão norteadora. Desta maneira surgiu a seguinte pergunta: quais as práticas/ condutas/ações interprofissionais que podem contribuir para a construção de um protocolo de assistência interprofissional às puérperas na APS?

A busca dos artigos foi realizada a partir das seguintes bases de dados: Literatura LatinoAmericana e do Caribe em Ciências da Saúde (LILACS), Excerpta Medica dataBASE (EMBASE), Web of Science, Cochrane Library e Medical Literature Analysis and Retrieval System Online (MEDLINE), no período novembro de 2018 a maio de 2019, utilizando os seguintes descritores com operador booleano: "Parient Care Team" AND "Clinical Protocols"; "Parient Care Team" AND "Postpartum Period"; "Postpartum Period" AND "Clinical Protocols"; "Parient Care Team" AND "Postpartum Period" AND "Clinical Protocols".

Foi utilizado o formulário padronizado CASP, para avaliação dos artigos encontrados na literatura. Os critérios analisados foram: 1) objetivo claro e justificado, 2) desenho metodológico qualitativo apropriado, 3) os procedimentos metodológicos são apresentados e discutidos, 4) seleção da amostra apropriada, 5) coleta de dados descrita, instrumentos explicitados, 6) a relação entre pesquisador e pesquisado é considerada, 7) cuidados éticos considerados, 8) análise dos dados rigorosa, 9) resultados claros, apresentados, discutidos, credibilidade dos achados, 10) validade dos achados, contribuições e implicações do conhecimento gerado pela pesquisa, bem como suas limitações (6).

Em relação à qualidade dos estudos e aos riscos de viés, aqueles com pelo menos 9 dos 10 itens propostos foram considerados com baixo risco de viés recebendo classificação A. Aqueles que atenderam entre 5 a 8 dos 10 itens propostos foram classificados como B, tendo alto risco de viés. Os estudos com pontuação abaixo de 5 foram excluídos da análise, bem como aqueles que não atenderam aos dois primeiros itens do formulário CASP $(6,7)$.

Após a execução dos critérios de elegibilidade do estudo, 36 artigos foram lidos na íntegra, destes 24 foram excluídos, pois não apresentavam cuidado multiprofissional voltado à puérpera. O fluxograma abaixo detalha a seleção dos estudos (figura 1). Inicialmente, dois pesquisadores 
Estratégias de cuidado interprofissional na assistência a saúde da puérpera na atenção primária à saúde. Uma revisão integrativa sobre prática clínica

independentes identificaram os potenciais estudos primários para inclusão e avaliação de qualidade dos mesmos.

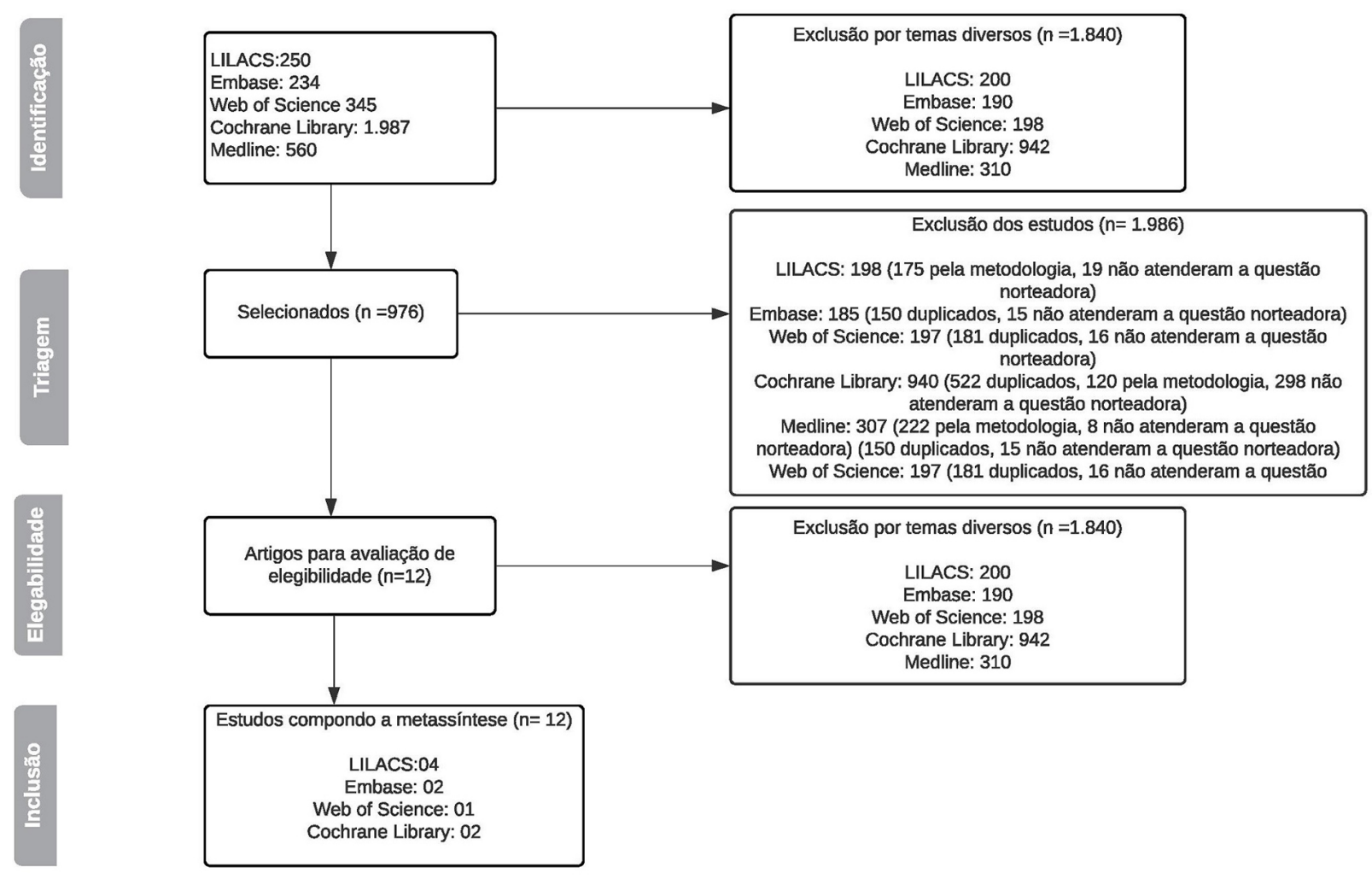

Figura 1. Fluxograma de seleção de estudos. Fortaleza, CE, 2019

Fuente: dados da pesquisa.

\section{Resultados}

Aplicaram-se os critérios CASP em 12 artigos, cujos resultados demonstraram que 05 obtiveram 09 ou 10 pontos; 07 ficaram entre 05 e 08 pontos. As demais características estão descritas na tabela 1 . 
Candice Feitosa de Alencar Mendes/ Ilziane Tomaz Ferreira/ Patrícia Freire de Vasconcelos/ Bianca Ianne Carlos Gonçalves/ et ai.

Tabela 1. Relação dos estudos segundo fonte, autor, título, tipo de estudo, população e CASP

\begin{tabular}{|c|c|c|c|c|c|}
\hline $\mathbf{N}^{\circ}$ & $\begin{array}{c}\text { Fonte } \\
\text { (Periódico) }\end{array}$ & Autores & $\begin{array}{l}\text { Tipo de } \\
\text { estudo }\end{array}$ & População estudada & $\begin{array}{l}\text { Nota } \\
\text { CASP }\end{array}$ \\
\hline 1 & $\begin{array}{l}\text { LILACS } \\
\text { (Australian } \\
\text { Journal of } \\
\text { Primary Health) }\end{array}$ & $\begin{array}{l}\text { Hartley et al. } \\
(2012)(8)\end{array}$ & $\begin{array}{l}\text { Estudo de } \\
\text { coorte }\end{array}$ & $\begin{array}{l}28 \text { mulheres no período } \\
\text { gravidico-puerperal }\end{array}$ & 07 \\
\hline 2 & $\begin{array}{l}\text { Medline (PLoS } \\
\text { One) }\end{array}$ & $\begin{array}{l}\text { Sitrin et al. (2013) } \\
(5)\end{array}$ & $\begin{array}{l}\text { Inquérito } \\
\text { transversal }\end{array}$ & 80 gestantes e 30 puérperas & 08 \\
\hline 3 & $\begin{array}{l}\text { Medline (BMC } \\
\text { Fam Pract) }\end{array}$ & $\begin{array}{l}\text { Brodribb, } \\
\text { Zadoroznyj, Dane } \\
(2013)(9)\end{array}$ & $\begin{array}{l}\text { Estudo } \\
\text { Qualitativo }\end{array}$ & $\begin{array}{l}88 \text { mães e seis médicos } \\
\text { generalistas }\end{array}$ & 08 \\
\hline 4 & $\begin{array}{l}\text { LILACS (Revista } \\
\text { Brasileira de } \\
\text { Enfermagem) }\end{array}$ & $\begin{array}{l}\text { Guerreiro et al. } \\
(2014)(10)\end{array}$ & $\begin{array}{l}\text { Estudo } \\
\text { descritivo, } \\
\text { qualitativo }\end{array}$ & 25 gestantes & 06 \\
\hline 5 & $\begin{array}{l}\text { Cochrane Library } \\
\text { (Rev enferm } \\
\text { UERJ) }\end{array}$ & $\begin{array}{l}\text { Mazzo, Brito, } \\
\text { Santos (2014) } \\
\text { (11) }\end{array}$ & $\begin{array}{l}\text { Estudo } \\
\text { qualitativo } \\
\text { e descritivo }\end{array}$ & 25 gestantes & 09 \\
\hline 6 & $\begin{array}{l}\text { Medline (Revista } \\
\text { Eletrônica Gestão } \\
\text { \& Saúde) }\end{array}$ & $\begin{array}{l}\text { Moura et al. } \\
(2014)(12)\end{array}$ & $\begin{array}{l}\text { Relato de } \\
\text { experiência }\end{array}$ & 55 puérperas & 10 \\
\hline 7 & $\begin{array}{l}\text { Cochrane Library } \\
\text { (Scand J Prim } \\
\text { Health Care) }\end{array}$ & $\begin{array}{l}\text { Munk-Olsen et al. } \\
(2015)(13)\end{array}$ & $\begin{array}{l}\text { Estudo de } \\
\text { coorte }\end{array}$ & $\begin{array}{l}939 \text { gestantes e } 1.436 \\
\text { puérperas }\end{array}$ & 09 \\
\hline 8 & $\begin{array}{l}\text { Web of Science } \\
\text { (Texto \& } \\
\text { Contexto } \\
\text { Enfermagem) }\end{array}$ & $\begin{array}{l}\text { Meira et al. } \\
(2015)(14)\end{array}$ & $\begin{array}{l}\text { Estudo } \\
\text { qualitativo- } \\
\text { descritivo }\end{array}$ & 16 profissionais de saúde & 07 \\
\hline 9 & $\begin{array}{l}\text { Embase (Revista } \\
\text { Gaúcha de } \\
\text { Enfermagem) }\end{array}$ & $\begin{array}{l}\text { Silva et al. (2016) } \\
(15)\end{array}$ & $\begin{array}{l}\text { Estudo } \\
\text { transversal }\end{array}$ & 190 puérperas & 08 \\
\hline 10 & $\begin{array}{l}\text { LILACS (Rev } \\
\text { Rene) }\end{array}$ & $\begin{array}{l}\text { Medeiros, Costa } \\
(2016)(16)\end{array}$ & $\begin{array}{l}\text { Pesquisa } \\
\text { qualitativa }\end{array}$ & $\begin{array}{l}16 \text { profissionais de saúde e } \\
20 \text { puérperas }\end{array}$ & 10 \\
\hline 11 & $\begin{array}{l}\text { LILACS (Revista } \\
\text { de Nutriçăo) }\end{array}$ & $\begin{array}{l}\text { Laporte-Pinfildi } \\
\text { et al. }(2016)(1)\end{array}$ & $\begin{array}{l}\text { Estudo } \\
\text { censitário }\end{array}$ & 38 profissionais de saúde & 08 \\
\hline 12 & $\begin{array}{l}\text { Embase } \\
\text { (Cadernos de } \\
\text { Saúde Pública) }\end{array}$ & $\begin{array}{l}\text { Corrêa et al. } \\
(2017)(4)\end{array}$ & $\begin{array}{l}\text { Pesquisa } \\
\text { qualitativa }\end{array}$ & $\begin{array}{l}\text { Equipe de saúde da família } \\
\text { (médica, enfermeira e cinco } \\
\text { agentes de saúde) e dez } \\
\text { gestantes }\end{array}$ & 10 \\
\hline
\end{tabular}

Fonte: dados da pesquisa.

$\mathrm{Na}$ análise dos estudos selecionados, observou-se que os cuidados às puérperas apresentados foram: visita domiciliar (4 artigos), assistência médica (2 artigos), assistência psicológica (2 artigos), educação em saúde (2 artigos), assistência nutricional (1 artigo) e acolhimento (1 artigo). A tabela 2 apresenta os principais cuidados e seus resultados dos estudos selecionados. 
Estratégias de cuidado interprofissional na assistência a saúde da puérpera na atenção primária à saúde. Uma revisão integrativa sobre prática clínica

Tabela 2. Cuidados prestados pelos profissionais e desfechos dos estudos selecionados

\begin{tabular}{|c|c|c|}
\hline $\mathrm{N}^{\circ}$ & Cuidado prestado & Principais resultados / Conclusões \\
\hline 1 & Assistência médica & $\begin{array}{l}\text { As mulheres valorizam a atenção primária, identificam questões que } \\
\text { facilitam e dificultam a comunicą̧ão entre pacientes e profissionais, } \\
\text { e descrevem bons encontros como aqueles em que se sentem } \\
\text { compreendidos, apoiados e tranquilizados. }\end{array}$ \\
\hline 2 & Visita domiciliar & $\begin{array}{l}\text { Alcançar mães e bebês com visitas domiciliares durante a gravidez e } \\
\text { dentro de três dias após o nascimento é alcançável usando os } \\
\text { sistemas de saúde comunitários existentes se os trabalhadores } \\
\text { estiverem disponiveis. }\end{array}$ \\
\hline 3 & Assistência médica & $\begin{array}{l}\text { As recomendaç̋̃es sobre as visitas ao médico dadas às mães } \\
\text { variaram de acordo com o setor de parto, obstetricia e modelo de } \\
\text { assistência à maternidade, resultando em desarticulação entre as } \\
\text { mães sobre o momento puerperal e o papel dos médicos no cuidado } \\
\text { de rotina no pós-parto. }\end{array}$ \\
\hline 4 & $\begin{array}{l}\text { Educação em } \\
\text { saúde }\end{array}$ & $\begin{array}{l}\text { As representaçōes das puérperas sobre a educação em saúde estão } \\
\text { ligadas às práticas educativas institucionais, com destaque para as } \\
\text { palestras, educação familiar e escolar, educação comunitária. }\end{array}$ \\
\hline 5 & Visita Domiciliar & $\begin{array}{l}\text { Durante a visita domiciliar percebeu-se pouca vigilància voltada } \\
\text { para a saúde da puérpera e as atençø̋es foram direcionadas, quase } \\
\text { que exclusivamente, para a criança. }\end{array}$ \\
\hline 6 & $\begin{array}{l}\text { Educaçẫo em } \\
\text { saúde }\end{array}$ & $\begin{array}{l}\text { As atividades de educação em saúde oportunizaram a troca de } \\
\text { saberes e de vivências, através de encontros em rodas de conversa. }\end{array}$ \\
\hline 7 & $\begin{array}{l}\text { Assistência } \\
\text { psicológica }\end{array}$ & $\begin{array}{l}\text { Mulheres com um episódio psiquiátrico pós-parto tiveram maior uso } \\
\text { de serviços de cuidados primários antes do parto, e neste grupo } \\
\text { específico de pacientes o próprio parto desencadeou um aumento } \\
\text { acentuado no número de consultas médicas no pós-parto. }\end{array}$ \\
\hline 8 & $\begin{array}{l}\text { Assistência } \\
\text { psicológica }\end{array}$ & $\begin{array}{l}\text { Os profissionais da atenção primária em saúde precisam ser } \\
\text { capacitados para identificar e tratar mulheres com depressão pós- } \\
\text { parto, bem como manuais e protocolos assistenciais precisam ser } \\
\text { estabelecidos para guiar a prática baseada em evidências cientificas. }\end{array}$ \\
\hline 9 & Visita domiciliar & $\begin{array}{l}\text { A participação do enfermeiro na visita estava associada à maior } \\
\text { realizaçăo de exame de mama, abdômen e investigação de condiçðes } \\
\text { emocionais. }\end{array}$ \\
\hline 10 & Visita domiciliar & $\begin{array}{l}\text { Imprescindivel por contribuir para a redução da morbimortalidade } \\
\text { da puérpera e recém-nascido, aumentar o vinculo entre unidade e } \\
\text { familia, e diminuir riscos de complicaçōes pós-parto, no entanto, a } \\
\text { atenção voltada a estas mulheres ainda está recoberta de grandes } \\
\text { desafios para alcançar uma assistência de fato satisfatória. }\end{array}$ \\
\hline 11 & $\begin{array}{l}\text { Assistência } \\
\text { nutricional }\end{array}$ & $\begin{array}{l}\text { A inserção do nutricionista foi o principal componente insuficiente } \\
\text { da dimensão estrutura, interferindo negativamente na assistência } \\
\text { nutricional na atenção básica. }\end{array}$ \\
\hline 12 & Acolhimento & $\begin{array}{l}\text { A incipiência do acolhimento mostra a necessidade de transformar } \\
\text { as práticas da equipe para conferir visibilidade à mulher, } \\
\text { qualificando o cuidado puerperal. }\end{array}$ \\
\hline
\end{tabular}

Fonte: dados da pesquisa.

A tabela 3 apresenta uma descrição das ações evidenciadas nos artigos, conforme a atuação de cada categoria pertencente a equipe multiprofissional e que condiz com o desenvolvimento de um serviço interdisciplinar. 
Candice Feitosa de Alencar Mendes/ Ilziane Tomaz Ferreira/ Patrícia Freire de Vasconcelos/ Bianca Ianne Carlos Gonçalves/ et ai.

Tabela 3. Condutas benéficas para assistência à puérpera na Atenção Primária desenvolvido pela Equipe Multiprofissional

\begin{tabular}{|c|c|c|}
\hline Atividade & Descrição & Realização \\
\hline $\begin{array}{l}\text { Acolhimento com } \\
\text { escuta qualificada }\end{array}$ & $\begin{array}{l}\text { Acolher as demandas da puérpera e sua familia } \\
\text { e identificar suas necessidades. }\end{array}$ & $\begin{array}{l}\text { Equipe } \\
\text { multiprofissional }\end{array}$ \\
\hline Avaliação global & $\begin{array}{l}\text { Entrevista, verificar documentação (Caderneta } \\
\text { das gestantes), verificar dados do RN e } \\
\text { identificar RN de risco, verificar fatores de } \\
\text { riscos, intercorrências. }\end{array}$ & $\begin{array}{l}\text { Equipe } \\
\text { multiprofissional }\end{array}$ \\
\hline $\begin{array}{l}\text { Exame fisico geral e } \\
\text { específico - puerpério } \\
\text { imediato e tardio }\end{array}$ & $\begin{array}{l}\text { Avaliar mamas e mamilo, examinar abdome, } \\
\text { examinar perineo e genitais externos. }\end{array}$ & $\begin{array}{l}\text { Enfermeiro (a) / } \\
\text { médico (a) }\end{array}$ \\
\hline $\begin{array}{l}\text { Abordagem e avaliação } \\
\text { nutricional }\end{array}$ & $\begin{array}{l}\text { Peso da mãe e do bebe, contraindicações } \\
\text { alimentares. }\end{array}$ & Nutricionista \\
\hline $\begin{array}{l}\text { Abordagem e avaliação } \\
\text { fisioterapêutica }\end{array}$ & $\begin{array}{l}\text { Orientação exercícios respiratórios, posturais e } \\
\text { de fortalecimento muscular, orientar quanto à } \\
\text { postura no leito, incentivar a deambulação } \\
\text { precoce e evitar posturas antálgicas. }\end{array}$ & Fisioterapeuta \\
\hline $\begin{array}{l}\text { Avaliação e condutas } \\
\text { psicossociais }\end{array}$ & $\begin{array}{l}\text { Contexto familiar e social, comorbidades } \\
\text { psicológicas. }\end{array}$ & Psicológicos \\
\hline $\begin{array}{l}\text { Plano de cuidado } \\
\text { materno }\end{array}$ & $\begin{array}{l}\text { Orientar, estimular e apoiar a familia na } \\
\text { amamentação exclusiva, reforçando a } \\
\text { importância e beneficios. Orientar cuidados } \\
\text { com as mamas. Compartilhar o cuidado - } \\
\text { encaminhar a puérpera aos serviços } \\
\text { especializados de saúde mental se detectado } \\
\text { sofrimento mental grave, apoiar a familia e } \\
\text { articular outras redes de apoio social, quando } \\
\text { necessário. Retirar os pontos da cesariana, } \\
\text { conforme orientação médica. Orientar quanto } \\
\text { ao retorno da atividade sexual e planejamento } \\
\text { reprodutivo. Orientar e recomendar métodos } \\
\text { contraceptivos de acordo com a preferência e } \\
\text { condiçåo clínica da mulher. }\end{array}$ & $\begin{array}{l}\text { Enfermeiro (a) / } \\
\text { médico (a) }\end{array}$ \\
\hline $\begin{array}{l}\text { Abordagem } \\
\text { medicamentosa }\end{array}$ & Uso de ferro e outros medicamentos. & Farmacêutico \\
\hline $\begin{array}{l}\text { Plano de cuidado com o } \\
\text { recém-nascido }\end{array}$ & $\begin{array}{l}\text { Imunização, Triagem Neonatal, Observar a } \\
\text { interação mãe-bebê,, Examinar RN, } \\
\text { Orientar manejos durante episódios de cólicas, } \\
\text { explicar as causas, orientar cuidado com coto } \\
\text { umbilical, orientar o banho de sol diário, } \\
\text { orientar e estimular ao acompanhamento de } \\
\text { puericultura. }\end{array}$ & $\begin{array}{l}\text { Equipe } \\
\text { multiprofissional }\end{array}$ \\
\hline
\end{tabular}

Fonte: dados da pesquisa. 
Estratégias de cuidado interprofissional na assistência a saúde da puérpera na atenção primária à saúde. Uma revisão integrativa sobre prática clínica

\section{Discussão}

No contexto da ESF, o profissional de saúde presta assistência às mulheres, de modo geral, com atividades relativas à saúde sexual e reprodutiva, do planejamento reprodutivo ao prénatal, câncer de colo uterino e de mamas, climatério, bem como ao período pós-parto. Essas atividades, segundo os estudos selecionados, foram desenvolvidas nas unidades e nas residências das usuárias, por meio de consultas, exames e visitas domiciliares. Este se evidencia como o método de disseminar o cuidado contínuo prestado à mulher no ciclo gravídico-puerperal (5-15). Na assistência em saúde da mulher há alguns grupos que se destacam como prioritários para o atendimento domiciliar. Tal é o caso da atenção às puérperas, devido a que o cuidado durante o puerpério deve ser imediato, individual e integral, estabelecendo um vínculo de confiança com a puérpera e toda sua família (10).

A visita domiciliar é compreendida como uma ferramenta estratégica de cuidado, e deve ser considerada característica do processo de trabalho das equipes de atenção básica (16). A atenção domiciliar está destinada a usuários que possuam problemas de saúde de difícil controle, e com dificuldade ou impossibilidade física de locomoção até uma unidade de saúde. Estes pacientes necessitam de cuidados com menor frequência e menor necessidade de recursos de saúde (11). O estudo apresentado por Medeiros e Costa (16) mostrou, através de relatos de enfermeiros, a importância da visita domiciliar relacionada a adaptação das mães com o bebê. Este processo facilita a consulta pós-parto, habitando as condições diárias de cada usuária. Além disso, a atenção primária tem um papel fundamental principalmente no processo educativo, pois contribui diretamente na promoção da saúde, bem como na prevenção de doenças comuns nesta fase.

Silva et al. (15) afirmam que o uso da visita domiciliar apresenta, em longo prazo, um efeito benéfico para a saúde do binômio, além de fortalecer o elo da família com o serviço de saúde. Trata-se, então, de um instrumento primordial para a práxis da equipe multiprofissional, no âmbito da ESF. Os profissionais da APS devem estar atentos e capacitados para apoiar a mulher e sua família; reconhecer as condições de risco e vulnerabilidade física, psíquica e social; e para utilizar os recursos das redes de saúde e intersetorial, lidando com os possíveis problemas surgidos nessa fase. Para isso, a equipe deve estar qualificada para desenvolver ações preconizadas na atenção à mulher no puerpério.

Moura et al. (12) realizaram atividade de educação em saúde com gestantes e puérperas. Tratase de uma ferramenta para promoção da saúde, e para o desenvolvimento de uma consciência crítica e reflexiva. Os temas abordados foram a importância do pré-natal, o desenvolvimento da gestação, as modificações psicológicas na gravidez, os cuidados com o corpo e as mamas, as alterações musculoesqueléticas e posturais na gestação, a atividade física na gestação, as infecções sexualmente transmissíveis, os tipos de parto, em especial o natural, o alívio da dor por meio da automassagem, e os cuidados com o recém-nascido e amamentação. Na ocasião, observou-se que a abordagem multidisciplinar proporcionou um atendimento integral à gestante, à medida que, ao se considerar os aspectos biopsicossociais, buscou-se a incorporação de novos saberes baseados em experiências já vividas. 
Candice Feitosa de Alencar Mendes/ Ilziane Tomaz Ferreira/ Patrícia

Freire de Vasconcelos/ Bianca Ianne Carlos Gonçalves/ et ai.

Laporte-Pinfildi et al. (1) expressaram que apenas 26,3\% das Unidades Básicas da Saúde e 33,3\% das Unidades de Saúde da Família apresentaram adequação, com relação ao espaço físico, e conformidade para realizar ações educativas e atividades em grupos. $\mathrm{O}$ atendimento pelo médico, de acordo com Hartley et al. (8) deve ser desempenhado com integralidade e equidade, alavancando uma importante contribuição no diagnóstico de agravos à saúde da puérpera e do neonato. Os autores identificaram questões que facilitam o atendimento, como o vínculo do profissional e paciente, a contextualização na comunidade, a frequência das consultas e visitas domiciliares. Igualmente, apontaram os aspectos que dificultam a atenção,como a elevada rotatividade do profissional no serviço, o interesse e disposição dos profissionais e a comunicação inócua entre pacientes e profissionais.

No estudo de Brodribb, Zadoroznyj e Dane (9) os médicos evidenciaram a necessidade de diretrizes consistentes para o envolvimento na rotina de atendimento pós-parto. Apesar da boa formação, essa carência preocupa aos gestores visando a qualidade do cuidado prestado as mulheres o mais precoce possível. Atrelado a isso, protocolos clínicos por meio da gestão da clínica apontam a minimizar a fragmentação do cuidado. Em outras palavras, estes favorecem a construção coletiva de padrões mais altos de qualidade e segurança do paciente (17). Episódios psiquiátricos são comumente associados ao puerpério pelos diversos fatores que infligem a mulher nessa fase (10). Em virtude disso, a assistência psicológica torna-se necessidade para a qualidade de vida do binômio mãe-bebê. Munk-Olsen et al. (13) conseguiram apresentar em sua pesquisa que o número de consultas com mães com depressão pós-parto é crescente e que esse trabalho deve ser desenvolvido desde a gestação, para que a efetividade da assistência seja aparente e integral.

Meira et al. (14) definiram como apenas fisiológicos os atendimentos em que os profissionais não estão adequados para desencadear uma atenção neurológica e psicológica que possa avaliar alterações emocionais relacionadas ao período puerperal. Ainda, destacam que os profissionais da APS precisam ser capacitados para identificar e tratar mulheres com depressão pós-parto, bem como construir manuais e protocolos assistenciais estabelecidos para guiar a prática baseada em evidências científicas. Laporte-Pinfildi et al. (1) evidenciaram importância da atuação do nutricionista na APS para assim garantir a integralidade do cuidado prestado pela equipe. Os parâmetros nutricionais e acompanhamento da composição corporal das puérperas e do bebê são essenciais para efetivar a atenção física destes (11). Nesse contexto, ao se consolidar os achados presentes nos estudos, observou-se que o cuidado pela equipe multiprofissional às puérperas proporciona conforto $\mathrm{e}$ liberdade de expressão das condições diárias. Isto se relaciona à realidade das mulheres em seus diversos aspectos da saúde e doença. $\mathrm{O}$ estudo encontrou limitações quanto à ausência de pesquisas que abordassem o cuidado multiprofissional e interdisciplinar, deixando evidente a necessidade e implementação de políticas que resguardem a atuação das categorias profissional e fomentem uma assistência de qualidade. 


\section{Conclusão}

Neste estudo, constatou-se que as evidências disponíveis para o cuidado à puérpera foram a visita domiciliar, educação em saúde e acolhimento como principais formas de abordagem e atenção às puérperas, bem como os diversos contextos de assistência à saúde como a médica, psicológica nutricional e farmacêutica. Evidenciou-se que há carência na orientação sobre esse período de mudanças na saúde da mulher, e que isso pode influenciar no cuidado a sua saúde e do recém-nascido.

Para fortalecer os conhecimentos e as práticas de autocuidado neste período, cada profissional deve reconhecer o cenário de apoio necessário ao binômio mãe-filho. Acredita-se que o uso da visita domiciliar como instrumento primordial, deve ser uma atuação de toda a equipe, desenvolvendo um trabalho interdisciplinar que contextualise principalmente o ponto de vista físico e mental.

Portanto, o cuidado interprofissional deve romper com a visão assistencialista, mecanicista do corpo, e apontar para o diálogo e a socialização de saberes e práticas entre profissionais e usuárias. Este processo deve incluir uma abordagem de educação em saúde, tendo a mãe como a sua protagonista, e valorizando o neonato, como membro ativo da sua condição de vida. No longo prazo isto deve apresentar efeitos benéficos às usuárias da atenção primária.

\section{Considerações éticas}

Nada no artigo infringe qualquer direito autoral ou de propriedade intelectual de outrem, pois caso contrário poderei responder integralmente por qualquer dano causado a terceiros, em todas as esferas administrativas e jurídicas cabíveis, nos estritos termos da Lei $n^{\circ}$ 9.610/98.

\section{Financiamiento}

Certificamos a participação na concepção do trabalho para tornar publica nossa responsabilidade pelo seu conteúdo, e que não omitimos quaisquer ligações ou acordos de financiamento entre os autores e companhias que possam ter interesse na publicação deste artigo.

\section{Conflito de interesse}

Declaramos que não temos nenhum conflito de interesse com o tema abordado.

\section{Referências}

1. Laporte-Pinfildi ASC, Zangirolani LTO, Spina N, Martins PA, Medeiros MAT. Atenção nutricional no pré-natal e no puerpério: percepção dos gestores da Atenção Básica à Saúde. Rev Nutr. 2016;29(1):109-123. http://www.scielo.br/pdf/rn/v29n1/1678-9865-rn-29-01-00109.pdf 
Candice Feitosa de Alencar Mendes/ Ilziane Tomaz Ferreira/ Patrícia

Freire de Vasconcelos/ Bianca Ianne Carlos Gonçalves/ et ai.

2. World Health Organization. Global Health Observatory (GHO) data. Women and health; 2017. h ttp://www.who.int/gho/en/

3. Tomazettia BM, Hermesb L, Martelloc NV, Schmittd PM, Braze MM, et al. A qualidade da assistência pré-natal sob olhar multiprofissional. Ciência\&Saúde. 2018;11(1):41-50. http://revistaseletronicas.p ucrs.br/ojs/index.php/faenfi/article/view/27078/16693.

4. Corrêa MSM, Feliciano KVO, Pedrosa EM, Souza AI. Acolhimento no cuidado à saúde da mulher no puerpério. Cad Saúde Pública. 2017;33(3):136-215. http://www.scielo.br/pdf/csp/v33n3/1678-4464 -csp-33-03-e00136215.pdf

5. Sitrin D, Guenther T, Murray J, Pilgrim N, Rubayet S, et al. Reaching mothers and babies with early postnatal home visits: The implementation realities of achieving high coverage in large-scale programs. PLOS ONE. 2013;8(7):12-19. https://www.ncbi.nlm.nih.gov/pmc/articles/PMC3714261/

6. Healthcare BV. Critical Appraisal Skills Programme (CASP); 2013. http://www.casp-uk.net/casp-tools -checklists

7. Costa AP. Processo de construção e avaliação de artigos de índole Qualitativa: possíveis caminhos? Rev Esc Enferm. USP. 2016;50(6):890-895. https://www.redalyc.org/articulo.oa?id=361049857002

8. Hartley S, Sutherland G, Brown S, Yelland J. 'You're more likely to tell the GP if you're asked': Women's views of care from general practitioners in the first postpartum year. Aust J Prim Health. 2012;18(4):308-312. https://www.ncbi.nlm.nih.gov/pubmed/22950969

9. Brodribb W, Zadoroznyj M, Dane A. The views of mothers and GPs about postpartum care in Australian general practice. BMC Fam Pract. 2013;25(14):139. https://www.ncbi.nlm.nih.gov/pmc/articles/PM C3851599/

10. Guerreiro EM, Rodrigues DP, Queiroz ABA, Ferreira MA. Educação em saúde no ciclo gravídico puerperal: sentidos atribuídos por puérperas. Rev Bras Enferm. 2014;67(1):13-21. http://www.sciel o.br/pdf/reben/v67n1/0034-7167-reben-67-01-0013.pdf

11. Mazzo MHSN, Brito RS, Santos FAPS. Atividades do enfermeiro durante a visita domiciliar pós-parto. Rev Enferm UERJ. 2014;22(5):663-667. http://www.facenf.uerj.br/v22n5/v22n5a13.pdf

12. Moura TNB, Santiago AKC, Santos MB, Lima ACG, Carvalho RB, et al. Educação em saúde como ferramenta para o cuidado à gestante, puérpera e recém-nascido: uma abordagem multidisciplinar. Rev Gest Saúde. 2014;6(1):657-666. https://dialnet.unirioja.es/servlet/articulo?codigo=5558848

13. Munk-Olsen T, Pedersen HS, Laursen TM, Fenger-Gron M, Vedsted P, et al. Use of primary health care prior to a postpartum psychiatric episode. Scand J Prim Health Care. 2015;33(2):127-133. https://w ww.ncbi.nlm.nih.gov/pmc/articles/PMC4834500/

14. Meira BM, Pereira PAS, Silveira MFA, Gualda DMR, Santos Júnior HPO. Desafios para profissionais da atenção primária no cuidado à mulher com Depressão Pós-Parto. Texto Contexto Enferm. 2015;24(3):706-712. http://www.scielo.br/pdf/tce/v24n3/pt_0104-0707-tce-24-03-00706.pdf. 
15. Silva LLB, Feliciano KVO, Oliveira LNFP, Pedrosa EN, Corrêa MSM, et al. Cuidados prestados à mulher na visita domiciliar da "Primeira Semana de Saúde Integral". Rev Gaúcha Enferm. 2016;37(3):e59248. http://dx.doi.org/10.1590/1983-1447.2016.03.59248

16. Medeiros LS, Costa ACM. Período puerperal: a importância da visita domiciliar para enfermeiros da Atenção Primária à Saúde. Rev Rene. 2016;17(1):112-119. http://periodicos.ufc.br/rene/article/view $/ 2622 / 2009$

17. Vasconcelos PF, Freitas CHA, Jorge MSB, Carvalho REF, Sousa Freire VEC, et al. Safety attributes in primary care: Understanding the needs of patients, health professionals, and managers. Public Health. 2019;171:31-40. https://www.sciencedirect.com/science/article/abs/pii/S0033350619301027 